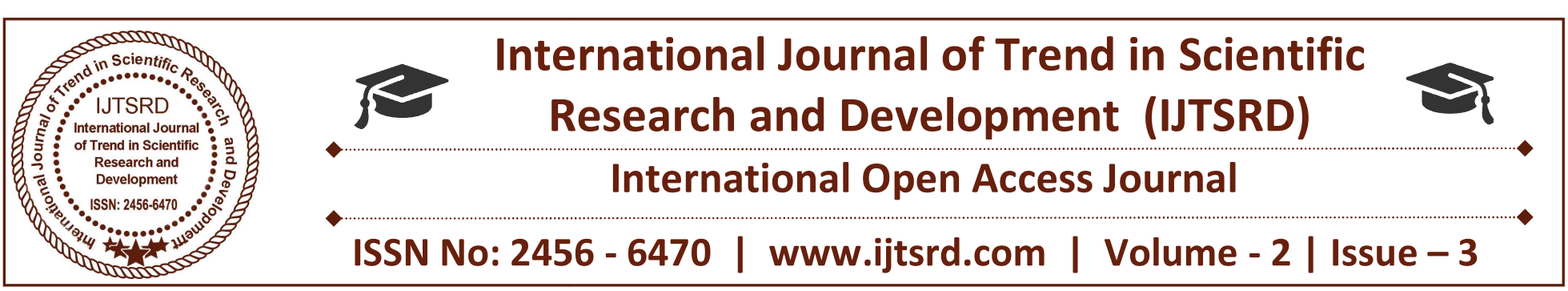

\title{
Life-Care Online Blood Donor Finder Application using Android
}

\author{
Pranali Dilip Kadam, Aniket Shivaji Vajire \\ Computer Technology, Bharati Vidyapeeth Institute of Technology, Kharghar, Maharashtra, India
}

\begin{abstract}
This paper introduce new technique to help people when they need blood. 'Life-care' is the android based mobile application, which uses the Java language. This Android app will help patients who are in need of Blood it will give the patient address of nearest Blood Bank along with information regarding Blood Banks. This app not only will connect Blood Banks but also willing Donors.
\end{abstract}

Keywords: Life care, Destination, Patient, Blood Bank

\section{Introduction}

The software system is an online blood bank management system that helps in managing various blood bank operations effectively. The project consists of a central repository containing various blood deposits available along with associated details. These details include blood type, storage area and date of storage. These details help in maintaining and monitoring the blood deposits. The project is an online system that allows to check weather required blood deposits of a particular group are available in the blood bank. Moreover the system also has added features such as patient name and contacts, blood booking and even need for certain blood group is posted on the website to find available donors for a blood emergency. This online system is developed on .net platform and supported by a Sql database to store blood and user specific details.

\section{PROPOSED SYSTEM}

In the stampede at Elphinstone Railway Station 27 people have been killed and scores are injured. The hospital was suffering from acute blood shortage. The hospital has asked the people donate blood. According to reports, there was a scarcity of A Negative, B Negative, and $A B$ Negative groups of hospital. This incident gives an idea about this "LIFE-CARE" app. This app is an online blood bank management system that helps in managing various blood bank operations effectively. This proposed system will find a blood donor from a specific area and provide the blood to the needful patient.

\section{Literature Survey}

The blood banks are available to provide blood to patient. We can demand blood with help of social media like, news, WhatsApp, Facebook etc.

To allow the probable recipients to make search and match the volunteer donors, and make request for the blood.

To provide a means for the blood bank to publicize and advertise blood donation programs.

\section{Interface}

For patient as well as donor GUI will be provided.

Patient interface must contain username, full name, password, confirm password, age, and blood group.

Donor interface will contain Username, password, age and Blood group.

\section{System Requirement}

Android, This word means a lot in present High-Tech World. Today Smartphone are known for its operating system which is Android. Earlier there is no option for operating systems like Android in mobile, as usual there are Symbian, java featured operating systems but today things had changed a lot, everyone wants a Smartphone which is functioned on Android only. In a very short span of time android created a reputed 
place in the market. What is this Android actually? Android is a software cluster for mobile devices that includes an operating system OS, key applications and middleware. The Android SDK provides the tools and APIs required to begin developing applications on the Android platform using the Java programming language. About the design, Kernel of Android is based on Linux kernel and further furnished by Google.

\section{Methodology}

There no Current method to find blood donor if there is no blood available in blood bank. Henceforth given product will provide way to search willing donor in $20 \mathrm{~km}$ radius of patient. Basic requirement for the product is to continuously update the database with donor new position. so patient will get most accurate result.

\section{A. Product Perspective}

\section{A.1 System Interfaces:}

System should provide functionality sign in for patient.

Along with sign in login functionality should be given Authentication of every user at every login attempt is necessary.

Patient will have search functionality.

\section{A.2 Interfaces:}

For patient as well as donor GUI will be provided.

Patient interface must contain username, full name, password, confirm password, age, and blood group.

Donor interface will contain Username, password, age and Blood group.

\section{A.3 Hardware Interfaces:}

Minimal requirement for hardware is to have Android smart phone with valid internet connection, gps tracking.

Minimum ram requirement is $400 \mathrm{mb}$.

\section{A.4 Software Interfaces:}

Specify the use of other required software products and interfaces with other application systems.

\section{A.5 Communications Interfaces:}

Socket programming is used.

Client server communication protocol is used.

\section{A.6 Memory Constraints}

Focus groups have determined that our target market has between 256-512M of RAM; therefore the design footprint should not exceed 256M.

\section{A.7 Operations}

1. Login

2. Sign In

3. Search Blood

4. Track gaps

5. Help

6. Background Task

\section{A.8 Assumptions and Dependencies}

This product will provide patient willing donors. The pathological testing is considered on part of patient. Donor can deny permission to donate blood. Donor is not obligated to donate blood.

\section{Aims and Objectives}

$>$ To allow the probable recipients to make search and match the volunteer donors, and make request for the blood

To provide a means for the blood bank to publicize and advertise blood donation programs.

To provide immediate storage and retrieval of data and information.

\section{Future Scope}

Online Chatting

Online backup of data in case if data is lost who is given blood and patient and donor details.

$>$ To provide immediate storage and retrieval of data and information.

\section{Conclusion}

Now a day's people are suffering from many diseases and accident in that case that if patient is in emergency situation that need blood it is very difficult to get blood that match with the patient's blood. Some blood group like "O-Negative", "AB-Negative" and so on is found in rare case. But with the help of this application we can easily find or get blood in emergency situation.

\section{Reference}

Following websites are referring to create this project Reports:

tools.android.com

http://www.codeproject.com 
http://androiddevelopers.blogspot.com

$>$ www.airpair.com

$>$ http://www.wikipedia.com

$>$ http://www.newboston.com/tutorials

$>\mathrm{http}: / /$ stackoverflow.com/documentation/android

\section{Books}

Following books and eBook are used to complete this

Project reports.

$>$ The Android Developer's Cookbook: Building

$>$ Applications with Android SDK

$>$ Bulletproof Android Practical Advice for Building

$>$ Secure Apps

$>$ Advanced Trigonometry by RD Sharma 